\title{
Comparison of Different Remission and Low Disease Definitions in Psoriatic Arthritis and Evaluation of Their Prognostic Value
}

\author{
Laura C. Coates, Alice B. Gottlieb, Joseph F. Merola, Caroline Boone, Annette Szumski, \\ and Amit Chhabra
}

ABSTRACT. Objective. There is no agreement on the optimal definitions for assessing disease state in patients with psoriatic arthritis (PsA), and some of the commonly used definitions do not include assessment of skin lesions. We investigated the performance of various definitions in patients with PsA and psoriasis.

Methods. This was a posthoc analysis of data from the PRESTA study. The remission definitions analyzed were very low disease activity (VLDA) index, defined as 7/7 of the minimal disease activity (MDA) cutoffs; Disease Activity Index for PsA (DAPSA); and clinical (c-) DAPSA. The low disease activity (LDA) definitions analyzed were as follows: MDA defined as 5/7 cutoffs; MDA joint with both the tender joint count (TJC) and swollen joint count (SJC) cutoffs mandated; MDA skin where skin cutoff was mandated; MDA joint + skin where TJC, SJC, and skin cutoffs were mandated; DAPSA LDA; and cDAPSA LDA.

Results. At Week 24, the proportions of patients achieving VLDA, DAPSA, and cDAPSA remission were $10 \%, 35 \%$, and $37 \%$, respectively. Of the patients achieving DAPSA and cDAPSA remission, $55 \%$ and $56 \%$, respectively, had Psoriasis Area and Severity Index $>1$. The proportions of patients achieving MDA 5/7, MDA skin, MDA joint, and MDA joint + skin were 44\%, 19\%, 36\%, and 14\%, respectively, versus $70 \%$ achieving DAPSA and cDAPSA LDA. Notable residual levels of psoriasis were observed in patients achieving the definitions that did not require skin disease control.

Conclusion. VLDA and MDA definitions are more stringent than DAPSA and CDAPSA definitions for the assessment of PsA. The relevance of residual disease to patients, however, remains to be determined. [Clinical Trial registration: ClinicalTrials.gov NCT00245960] (First Release October 152018 ; J Rheumatol 2019;46:160-5; doi:10.3899/jrheum.180249)

Key Indexing Terms:

PSORIATIC ARTHRITIS DISEASE ACTIVITY ETANERCEPT REMISSION

From the Botnar Research Centre, University of Oxford, Oxford, UK; New York Medical College at Metropolitan Hospital, New York, New York, USA; Harvard Medical School, Brigham and Women's Hospital, Boston, Massachusetts, USA; Pfizer, Brussels, Belgium; inVentiv Health, Princeton, New Jersey, USA; Pfizer, New York, New York, USA.

This study was funded by Pfizer. L.C. Coates is funded by a UK National Institute for Health Research (NIHR) Clinician Scientist award. The research was supported by the NIHR Oxford Biomedical Research Centre. The views expressed are those of the author $(s)$ and not necessarily those of the UK National Health Service, the NIHR, or the UK Department of Health. Medical writing support was provided by Sabrina Giavara, PhD, of Engage Scientific Solutions, and was funded by Pfizer.

$L C C$ has received fees from AbbVie, Novartis, and UCB. ABG has received fees from Janssen Inc., AbbVie, UCB, Novartis, and Eli Lilly. JFM is a consultant and/or investigator for Merck Research Laboratories, AbbVie, Eli Lilly and Co., Novartis, Janssen, UCB, Samumed, Celgene, Sanofi Regeneron, GSK, Almirall, Sun Pharma, Biogen, Pfizer, Incyte, Aclaris, and Leo Pharma. AS is an employee of inVentiv and was contracted by Pfizer in connection with the development of the manuscript. AC is an employee of Pfizer. CB was employed by Pfizer at the time of the study. L.C. Coates, MBChB, PhD, NIHR Clinician Scientist, Botnar Research Centre, University of Oxford; A.B. Gottlieb, MD, PhD, Professor of Dermatology, Department of Dermatology, New York Medical College, Metropolitan Hospital; J.F. Merola, MD, MMSc, Brigham and Women's Hospital, Harvard Medical School; C. Boone, PharmD, (formerly) Medical Manager, Pfizer Innovative Health, Medical Department, Pfizer, Brussels, at the time of the study; A. Szumski, MS, Principal Statistician,
inVentiv Health; A. Chhabra, MBBS, MPH, Senior Medical Director Pfizer, New York.

Address correspondence to L.C. Coates, Botnar Research Centre, Windmill Road, Oxford OX3 7LD, UK.

E-mail: laura.coates@ndorms.ox.ac.uk

Accepted for publication June 28, 2018

Psoriasis is a skin disorder characterized by scaly and itchy plaques that affects $1-3 \%$ of the world's population ${ }^{1,2}$. Up to $40 \%$ of people with psoriasis develop psoriatic arthritis $(\mathrm{PsA})^{3,4,5}$, a form of inflammatory arthritis affecting the joints and the tendons around the joints, which is characterized by specific manifestations such as the swelling of whole digits (dactylitis) and the inflammation of the entheses (enthesitis). The prevalence of PsA in the general population is about $0.1-1.0 \%{ }^{3}$. PsA usually appears in patients with psoriasis within 10-12 years, and patients with extensive psoriatic skin lesions are at increased risk of developing PsA ${ }^{1,3,6}$. Patients with both PsA and psoriasis tend to have a decreased quality of life compared with patients with psoriasis only ${ }^{7,8}$, owing to the discomfort caused by the skin condition as well as the pain and disability caused by the joint damage. 
A wide range of treatments is available for PsA, including nonsteroidal antiinflammatory drugs, conventional synthetic disease-modifying antirheumatic drugs (DMARD), interleukin (IL)-12/23, IL-17A, phosphodiesterase type 4 (PDE4), and tumor necrosis factor- $\alpha$ (TNF- $\alpha$ ) inhibitors $9,10,11,12$. Several studies have shown that clinical remission or minimal disease activity (MDA) can be achieved by $40-60 \%$ of patients using TNF- $\alpha$ inhibitors ${ }^{13,14,15,16}$. In addition, IL-12/23, IL-17A, and PDE4 inhibitors are valid alternatives to DMARD therapy ${ }^{11,17,18,19}$.

Adoption of a treat-to-target (T2T) approach aiming at tight disease control has been shown to improve physician- and patient-reported outcomes in patients with recent-onset PsA ${ }^{20}$; therefore, European League Against Rheumatism guidelines suggest adopting a T2T approach as the standard of care for patients with PsA, with either remission or low disease activity (LDA) as the target ${ }^{9}$.

The many different manifestations associated with PsA pose a challenge when assessing patients and their response to treatments. This has led to the development of several composite definitions encompassing clinically important aspects of the disease such as arthritis, psoriasis, enthesitis, pain, patient-assessed global disease activity, and physical function, which then combine into a single disease activity score $^{21}$. One such composite definition is the Disease Activity Index for PsA (DAPSA), which was adapted from the Disease Activity Index for Reactive Arthritis and validated using data from PsA clinical trials ${ }^{22}$. DAPSA cutoffs for remission and LDA have been established; however, DAPSA focuses primarily on peripheral arthritis, and does not include other PsA manifestations such as psoriasis or enthesitis. In 2010, the MDA criteria were developed to encompass most PsA domains ${ }^{13}$. Remission or very low disease activity (VLDA) is achieved when 7 of the 7 MDA cutoffs are met, while meeting only 5/7 cutoffs is classified as a minimal or low disease state ${ }^{23}$. According to recent recommendations by an international task force, either DAPSA or MDA should be used to define the target in disease control; however, further research is needed to establish the pros and cons of each of these definitions in PsA assessment ${ }^{24}$.

The objective of our present analysis was to investigate the performance of various remission and LDA definitions in patients with PsA with extensive skin lesions.

\section{MATERIALS AND METHODS}

This was a posthoc analysis of data from the PRESTA ${ }^{25}$ double-blind multicenter clinical study (NCT00245960), in which patients with both psoriasis and PsA were randomized to receive $50 \mathrm{mg}$ etanercept (ETN) either twice weekly (BIW) or once weekly (QW) for 12 weeks by subcutaneous injection ${ }^{25}$. This was followed by an additional 12 weeks in which both cohorts received open-label ETN $50 \mathrm{mg}$ QW. An independent ethics committee or institutional review board approved the protocol of this study $^{25}$. All participants provided a signed informed consent form ${ }^{25}$.

Definition of remission and LDA. The remission and LDA definitions evaluated in this analysis are shown in Table 1. Briefly, VLDA remission was defined as meeting $7 / 7$ cutoffs of the MDA definition [tender joint count $(\mathrm{TJC}) \leq 1$, swollen joint count $(\mathrm{SJC}) \leq 1$, Psoriasis Area and Severity Index (PASI) $\leq 1$, patient's global assessment visual analog scale (PtGA VAS) $\leq 20 \mathrm{~mm}$, Pt pain VAS $\leq 15 \mathrm{~mm}$, Health Assessment Questionnaire (HAQ) $\leq 0.5$, tender entheseal points $\leq 1]$. DAPSA remission was defined as DAPSA $\leq 4$ [TJC, SJC, PtGA VAS, Pt pain VAS, C-reactive protein (CRP)]; cDAPSA (clinical DAPSA without CRP) remission was defined as cDAPSA $\leq 4$. The LDA definitions evaluated were DAPSA LDA $\leq 14$ (TJC, SJC, PtGA VAS, Pt pain VAS, CRP); cDAPSA LDA $\leq 13$; MDA defined as 5/7 cutoffs; MDA joint with both TJC and SJC cutoffs mandated, and with any $3 / 5$ of the remaining cutoffs; MDA skin where PASI cutoff was mandated, and with any $4 / 6$ of the remaining cutoffs; and MDA joint + skin where the TJC, SJC, and skin cutoffs were mandated, and with any $2 / 4$ of the remaining cutoffs.

Statistical analysis. The proportion of patients achieving remission and LDA responses was calculated at Week 12 and at Week 24. Treatment effect was calculated using chi-square tests. Response was compared by analyzing the levels of concordance/discordance and by assessing the frequency of occurrence of the different levels of residual disease (e.g., $>0,>1$ ) across the PsA manifestations (dactylitis, enthesitis, psoriasis, TJC, SJC) among the definitions. Levels of residual disease were considered clinically meaningful if they were above the MDA/LDA cutoffs. Total discordance was calculated as the sum of 2 individual discordances (i.e., the percentage of patients who had a VLDA but not DAPSA or cDAPSA remission response, plus the percentage of patients who did not have a VLDA response but had a DAPSA or cDAPSA remission response). Level of agreement was tested by $\kappa$ coefficients calculated for each pair of definitions. $\kappa$ values $<0$ indicate no agreement, 0-0.20 slight agreement, 0.21-0.40 fair agreement, 0.41-0.60 moderate agreement, $0.61-0.80$ substantial agreement, and $0.81-1.0$ almost perfect agreement ${ }^{26}$

\section{RESULTS}

PRESTA baseline characteristics. The PRESTA study included 752 patients with PsA and psoriasis at baseline. The mean age was 46.5 years, $62.9 \%$ were male, the mean duration of psoriasis was 18.9 years, and the mean duration of PsA was 7.0 years $^{25}$. A total of 379 patients received ETN $50 \mathrm{mg}$ BIW and 373 patients received ETN $50 \mathrm{mg}$ QW.

Remission. At Week 24, a greater proportion of patients achieved DAPSA $(232 / 669,35 \%)$ or cDAPSA $(251 / 676$, $37 \%)$ remission than VLDA remission (67/669 and 68/676, both $10 \%)$. Figure $1 \mathrm{~A}-\mathrm{B}$ shows the proportion of patients achieving remission by treatment cohort. Discordance between VLDA and DAPSA remission was 25\% $(\kappa=0.33)$, while discordance between VLDA and cDAPSA remission was $27 \%$ ( $\kappa=0.32$ ). Only $10 \%$ of patients achieving DAPSA but not VLDA remission were in discordance with patients achieving MDA 5/7 ( $\kappa$ not available due to all patients having the same observation). Two patients $(0.3 \%)$ did not achieve VLDA remission solely because of a HAQ score $>0.5$; in contrast, 15/232 (6\%) and 17/251 (7\%) patients with HAQ score $>0.5$ were among patients who achieved DAPSA and cDAPSA remission, respectively.

Two patients $(0.3 \%)$ who achieved VLDA remission did not achieve DAPSA remission and 167/669 (25\%) who achieved DAPSA remission did not achieve VLDA remission. No patients achieved VLDA remission but not cDAPSA remission, while 183/676 (27\%) achieved cDAPSA

Personal non-commercial use only. The Journal of Rheumatology Copyright @ 2019 . All rights reserved. 
Table 1. Remission and low disease activity definitions investigated.

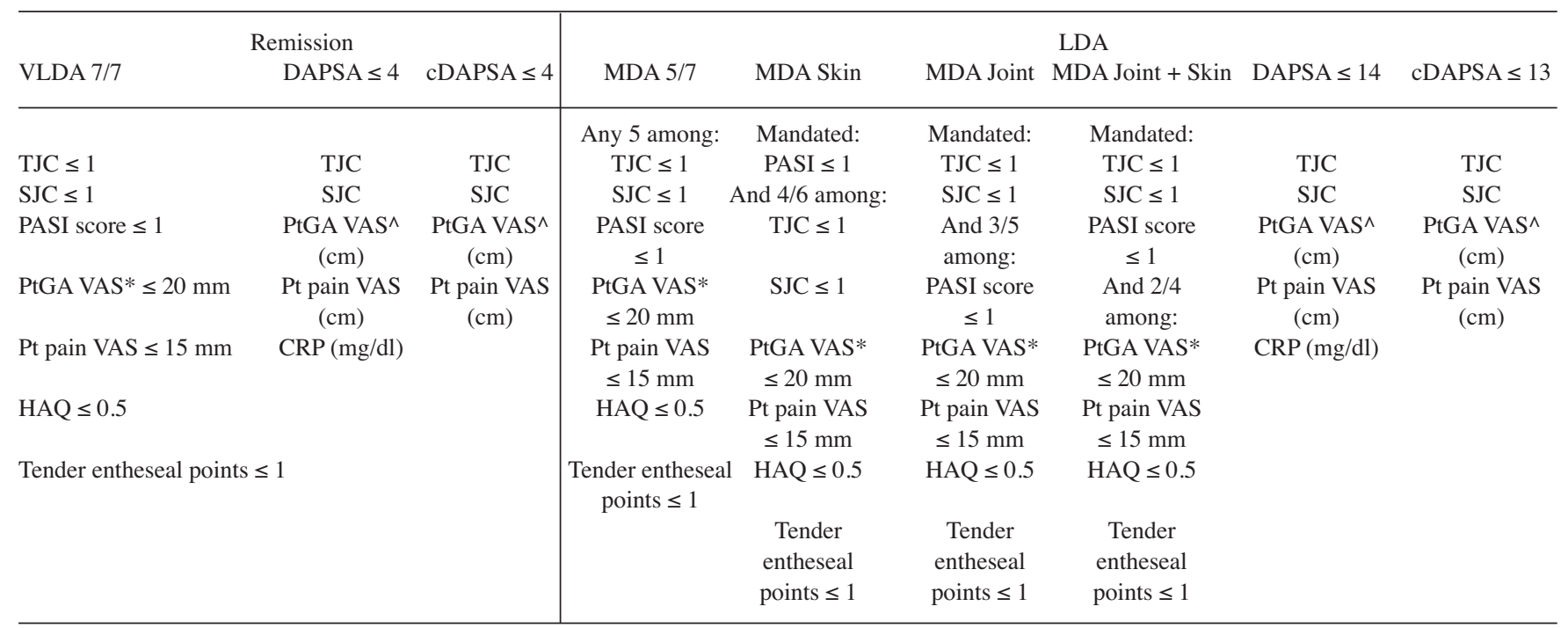

* PtGA VAS of the maximum component either of psoriasis activity (0-100) or of arthritis activity (0-100). ^ PtGA VAS of arthritis activity (0-100)/10. LDA: low disease activity; VLDA: very LDA; DAPSA: Disease Activity Index for Psoriatic Arthritis; cDAPSA: clinical DAPSA; MDA: minimal disease activity; TJC: tender joint count; SJC: swollen joint count; PASI: Psoriasis Area and Severity Index; PtGA VAS: patient visual analog scale; HAQ: Health Assessment Questionnaire; CRP: C-reactive protein.

A

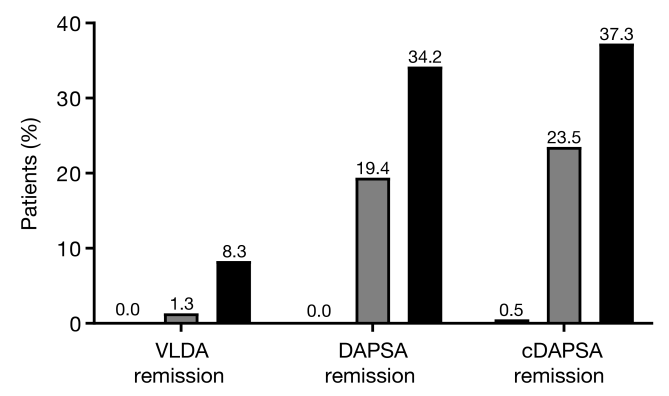

C

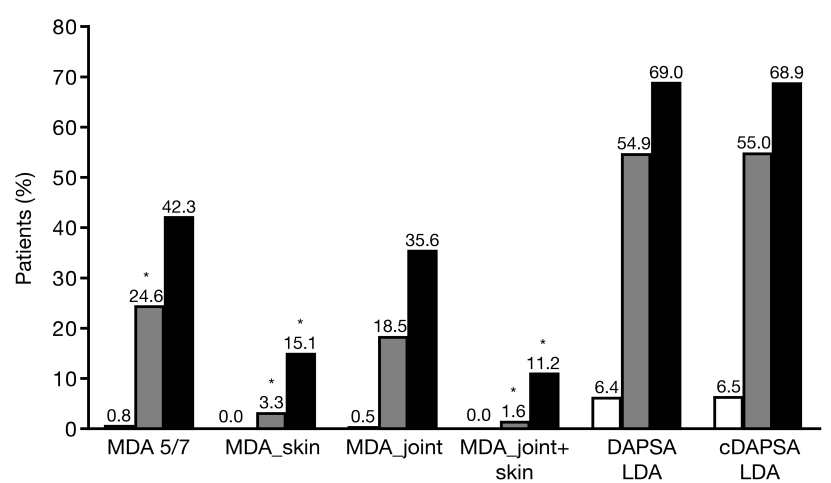

B

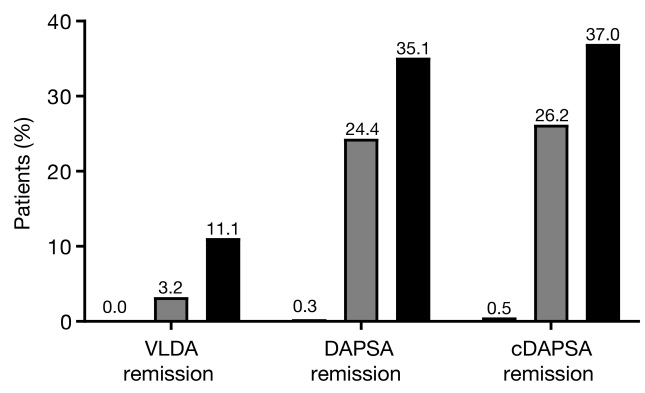

D

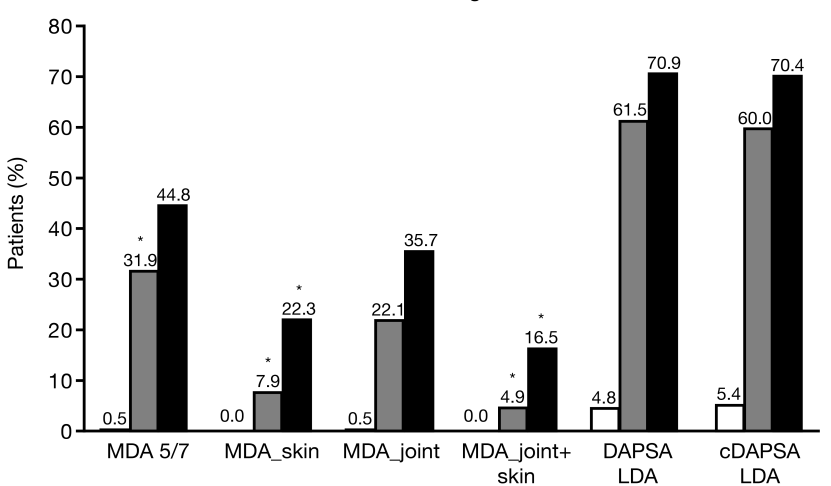

Figure 1. Proportion of patients achieving each definition in the 2 treatment groups. A. Patients receiving ETN $50 \mathrm{mg}$ QW/QW achieving remission. B. Patients receiving ETN $50 \mathrm{mg}$ BIW/QW achieving remission. C. Patients receiving ETN $50 \mathrm{mg}$ QW/QW achieving LDA. D. Patients receiving ETN $50 \mathrm{mg}$ BIW/QW achieving LDA. * $\mathrm{p}<0.05$ indicates a significant difference between ETN $50 \mathrm{mg}$ QW and ETN $50 \mathrm{mg}$ BIW within the LDA definition specified. ETN: etanercept; QW: once weekly; BIW: twice weekly; LDA: low disease activity; VLDA: very LDA; DAPSA: Disease Activity Index for Psoriatic Arthritis; cDAPSA: clinical DAPSA; MDA: minimal disease activity. 
remission but not VLDA remission. Frequency of residual disease in the 167 patients achieving DAPSA but not VLDA remission is provided in Table 2. Mild to moderate residual psoriasis (PASI 1-9) was found in $145 / 167$ patients (87\%) while 14/167 patients (8\%) had a high level of residual psoriasis (PASI $\geq 10$ ). Further, $15 / 166$ patients $(9 \%)$ had a HAQ score $>0.5$. In contrast, the only 2 patients achieving VLDA but not DAPSA remission had no or little residual disease across the different cutoffs, but 1 patient had a slightly raised level of CRP $(3.32 \mathrm{mg} / \mathrm{dl})$, giving a total DAPSA score of 6.32 (DAPSA score for the other patient achieving VLDA but not DAPSA remission was 4.12).

Residual levels of dactylitis and enthesitis at Week 24 were similar across all definitions (all $\leq 2.9 \%$; Table 2 ). However, high proportions of patients with PASI $>1$ were among patients achieving DAPSA and cDAPSA remission (Table 2). Raised CRP levels (upper limit of normal $>8.99$ $\mathrm{mg} / \mathrm{dl}$ ) were found in $8 \%, 6 \%$, and $9 \%$ of patients in the VLDA, DAPSA, and cDAPSA remission groups, respectively (Table 2).

$L D A$. At Week 12, a statistically significant difference $(\mathrm{p}<0.05)$ was observed between the $50 \mathrm{mg}$ ETN QW and BIW cohorts in the proportion of patients achieving LDA using the definitions that required a skin cutoff (MDA skin and MDA joint + skin) and MDA 5/7, but not DAPSA LDA, cDAPSA LDA, or MDA joint (Figure $1 \mathrm{C}-\mathrm{D}$ ). At Week 24, a significant difference $(p<0.05)$ between the 2 ETN treatment regimens was observed in the cohort of patients using the MDA skin and MDA joint + skin definitions (Figure $1 \mathrm{C}-\mathrm{D})$. At the end of the study, LDA was achieved by $44 \%$ of patients in MDA 5/7, 19\% in MDA skin, 36\% in MDA joint, and $14 \%$ in MDA joint + skin, versus $70 \%$ in DAPSA and cDAPSA LDA. The highest proportion of discordance in treatment response was observed between MDA skin or MDA joint + skin and DAPSA LDA or cDAPSA LDA (Table 3). Fewer than $2 \%$ of patients achieving any MDA definition did not achieve DAPSA or cDAPSA LDA, while
$27-56 \%$ of patients who achieved DAPSA or cDAPSA LDA did not achieve the corresponding MDA definition (all $\kappa<0.5$, Table 3 ).

The levels of residual disease across the different LDA definitions are shown in Table 4. The majority of patients had no residual arthritis, although levels were numerically higher in the DAPSA definitions, while notable residual levels of psoriasis were observed in the LDA definitions that did not require skin disease control. Patients achieving MDA joint + skin $(11 \%$ in the ETN $50 \mathrm{mg}$ QW/QW cohort, and 17\% in the ETN $50 \mathrm{mg}$ BIW/QW cohort at Week 24) had the lowest levels of residual disease across all cutoffs.

\section{DISCUSSION}

Our posthoc analysis of data from the PRESTA study has shown that only a small percentage of patients achieved VLDA remission, in contrast to the proportion of patients achieving DAPSA and cDAPSA remission. In addition, patients achieving VLDA had better disease control in all clinical aspects, thus making VLDA remission a more stringent definition than either DAPSA or cDAPSA remission. As a consequence, VLDA remission target might not be easily achieved in current clinical practice. However, VLDA has the advantage of including a measurement for psoriasis; thus, it might be more clinically meaningful than DAPSA or cDAPSA definitions in assessing remission in patients with PsA and extensive skin lesions. When looking at levels of residual disease in patients achieving DAPSA but not VLDA remission, residual psoriasis was observed in $49 \%$ of patients. In contrast, the 2 patients achieving VLDA but not DAPSA remission had no notable levels of residual disease and the high DAPSA remission score in 1 of these patients was due to raised levels of CRP, which may indicate presence of concomitant illnesses. CRP is considered a marker of inflammation in several conditions, including psoriasis and rheumatoid arthritis, and high baseline levels of CRP have been found in some patients with psoriasis with or without

Table 2. Proportion of patients with residual disease at Week 24 across each remission definition.

\begin{tabular}{|c|c|c|c|c|}
\hline Variables & VLDA Remission & DAPSA Remission & cDAPSA Remission & $\begin{array}{c}\text { DAPSA Remission+ VLDA } \\
\text { Remission-* }\end{array}$ \\
\hline Dactylitis (1-3) & $2 / 68(2.9)$ & $5 / 231(2.2)$ & $5 / 250(2.0)$ & $3 / 166(1.8)$ \\
\hline HAQ score $>0.5$ & $0 / 68(0.0)$ & $15 / 231(6.5)$ & $17 / 250(6.8)$ & $15 / 166(9.0)$ \\
\hline PASI score 0 & $47 / 68(69.1)$ & $54 / 232(23.3)$ & $58 / 251(23.1)$ & $8 / 167(4.8)$ \\
\hline PASI score 1 & $21 / 68(30.9)$ & $50 / 232(21.6)$ & $53 / 251(21.1)$ & $31 / 167(18.6)$ \\
\hline $\mathrm{SJC}>1$ & $0 / 68(0.0)$ & $7 / 232(3.0)$ & $9 / 251(3.6)$ & $7 / 167$ (4.2) \\
\hline $\mathrm{TJC}>1$ & $0 / 68(0.0)$ & $7 / 232(3.0)$ & $9 / 251(3.6)$ & $7 / 167(4.2)$ \\
\hline CRP $($ ULN > $8.99 \mathrm{mg} / \mathrm{dl})$ & $5 / 67(7.5)$ & $14 / 232(6.0)$ & $22 / 248(8.9)$ & $10 / 167(6.0)$ \\
\hline
\end{tabular}

Values are $\mathrm{n} / \mathrm{N}(\%)$. * Frequency of residual disease for patients who met DAPSA but not VLDA remission at Week 24. VLDA: very low disease activity; DAPSA: Disease Activity for Psoriatic Arthritis; cDAPSA: clinical DAPSA; HAQ: Health Assessment Questionnaire; PASI: Psoriasis Area and Severity Index; SJC: swollen joint count; TJC: tender joint count; CRP: C-reactive protein; ULN: upper limit of normal.

Personal non-commercial use only. The Journal of Rheumatology Copyright $@$ 2019. All rights reserved 
Table 3. Proportion of patients in discordance for each pair of LDA definitions at Week 24.

\begin{tabular}{|c|c|c|c|c|}
\hline LDA Definition 1 & LDA Definition 2 & $\begin{array}{l}\text { Proportion of Patients Achieving } \\
\text { LDA } 2 \text { but Not LDA } 1, \mathrm{n} / \mathrm{N}(\%)\end{array}$ & $\begin{array}{l}\text { Proportion of Patients Achieving } \\
\text { LDA } 1 \text { but Not LDA 2, n/N (\%) }\end{array}$ & к Coefficient* \\
\hline MDA $5 / 7$ & DAPSA LDA & $184 / 669(27.5)$ & $10 / 669(1.5)$ & 0.45 \\
\hline MDA joint & DAPSA LDA & $225 / 668(33.7)$ & $1 / 665(0.1)$ & 0.39 \\
\hline MDA joint + skin & DAPSA LDA & $373 / 669(55.8)$ & $1 / 669(0.1)$ & 0.13 \\
\hline MDA $5 / 7$ & cDAPSA LDA & $183 / 676(27.1)$ & $10 / 676(1.5)$ & 0.45 \\
\hline MDA joint + skin & cDAPSA LDA & $375 / 676(55.5)$ & $1 / 676(0.1)$ & 0.13 \\
\hline
\end{tabular}

* From $\kappa$ test of agreement between LDA definition 1 and definition 2. LDA: low disease activity; MDA: minimal disease activity; DAPSA: Disease Activity for Psoriatic Arthritis; cDAPSA: clinical DAPSA.

Table 4. Proportion of patients with residual disease across each LDA definition at Week 24.

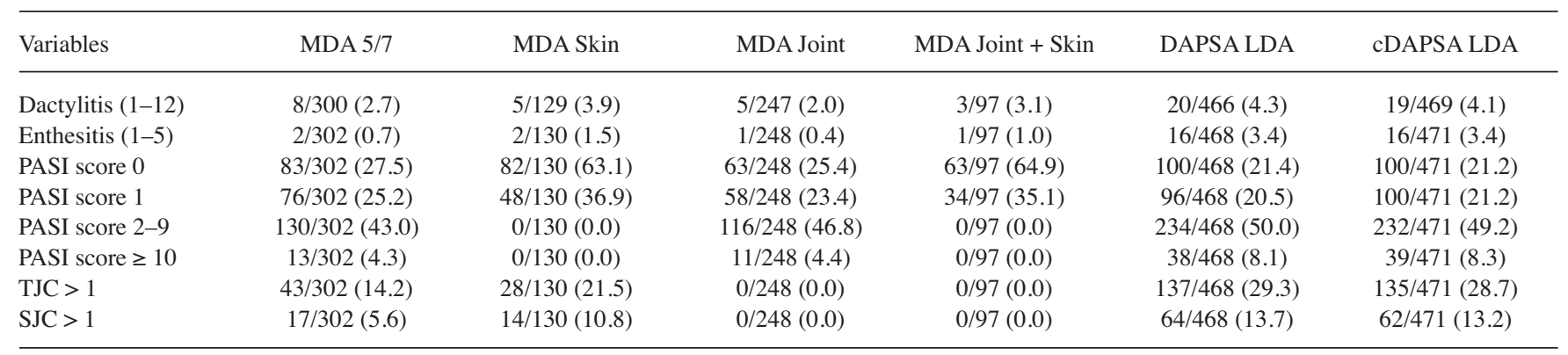

Values are n/N (\%). LDA: low disease activity; MDA: minimal disease activity; DAPSA: Disease Activity for Psoriatic Arthritis; cDAPSA: clinical DAPSA; PASI: Psoriasis Area and Severity Index; TJC: tender joint count; SJC: swollen joint count.

$\mathrm{PsA}^{27}$. In our analysis, the proportion of patients with raised CRP levels was similar across all definitions and did not appear to provide further information on current disease activity level. Thus, we propose that a laboratory marker to facilitate disease assessment in clinical practice may not be mandatory.

Overall, fewer patients achieved LDA in the MDA definitions than in DAPSA or cDAPSA definitions. The highest proportion of discordance between MDA and DAPSA or cDAPSA was observed in the definitions that required measurement of skin disease. When looking at levels of residual disease in patients achieving DAPSA or cDAPSA LDA, levels of residual psoriasis were high; thus inclusion of a skin cutoff might be required in the assessment of patients with PsA and psoriasis. Residual arthritis also appeared higher in patients achieving DAPSA or cDAPSA LDA than in patients achieving any other MDA definition, which indicates that MDA cutoffs are more demanding than those in DAPSA or cDAPSA LDA. This may be because 1 element within DAPSA may be high, but if the other items are low, then LDA is still achieved. In contrast, MDA assesses each item individually. Among the different MDA definitions, MDA joint + skin gave the lowest proportion of patients with residual disease activity across all domains but was achieved by only a few patients. This suggests that, under current disease management, achieving LDA in both joint and skin domains may be difficult in most patients presenting with severe skin and joint disease. Currently, it is unclear whether less residual disease improves physical function and decreases radiographic damage; therefore, it is not possible to conclude which definition leads to better clinical outcomes $^{28,29}$. However, a recent study showed that better "body functions" and "activity" were achieved by patients in both MDA and DAPSA LDA rather than in DAPSA LDA only ${ }^{30}$.

Our posthoc analysis showed that there were lower levels of residual disease in patients achieving VLDA remission compared with those in patients achieving DAPSA or cDAPSA remission. Thus, VLDA cutoffs are more demanding than DAPSA or cDAPSA definitions for the assessment of remission in PsA, resulting in some level of disagreement among these definitions. In addition, when skin disease control is not included in a treatment target for PsA, notable levels of psoriasis are missed. Therefore, adoption of a composite definition that includes skin assessment might be desirable in patients with PsA and extensive skin lesions. Because cutoffs in these definitions are more stringent than those in DAPSA or cDAPSA definitions, fewer patients will attain remission or MDA under current disease management, but may receive more comprehensive PsA treatment. Our 
findings may assist physicians in choosing a valid composite definition that facilitates the assessment and treatment of patients with PsA and psoriasis in clinical practice.

\section{ACKNOWLEDGMENT}

We thank all patients who participated in the trial and medical staff of all participating centers.

\section{REFERENCES}

1. Chang CA, Gottlieb AB, Lizzul PF. Management of psoriatic arthritis from the view of the dermatologist. Nat Rev Rheumatol 2011;7:588-98.

2. Kurd SK, Gelfand JM. The prevalence of previously diagnosed and undiagnosed psoriasis in US adults: results from NHANES 2003-2004. J Am Acad Dermatol 2009;60:218-24.

3. Gladman DD, Antoni C, Mease P, Clegg DO, Nash P. Psoriatic arthritis: epidemiology, clinical features, course, and outcome. Ann Rheum Dis 2005;64 Suppl 2:ii14-7.

4. Gottlieb A, Korman NJ, Gordon KB, Feldman SR, Lebwohl M, Koo JY, et al. Guidelines of care for the management of psoriasis and psoriatic arthritis: Section 2. Psoriatic arthritis: overview and guidelines of care for treatment with an emphasis on the biologics. J Am Acad Dermatol 2008;58:851-64.

5. Stolwijk C, Boonen A, van Tubergen A, Reveille JD. Epidemiology of spondyloarthritis. Rheum Dis Clin North Am 2012;38:441-76.

6. Gottlieb AB, Mease PJ, Mark Jackson J, Eisen D, Amy Xia H, Asare $\mathrm{C}$, et al. Clinical characteristics of psoriatic arthritis and psoriasis in dermatologists' offices. J Dermatolog Treat 2006;17:279-87.

7. Mease PJ, Menter MA. Quality-of-life issues in psoriasis and psoriatic arthritis: outcome measures and therapies from a dermatological perspective. J Am Acad Dermatol 2006;54:685-704.

8. Lundberg L, Johannesson M, Silverdahl M, Hermansson C, Lindberg M. Health-related quality of life in patients with psoriasis and atopic dermatitis measured with SF-36, DLQI and a subjective measure of disease activity. Acta Derm Venereol 2000;80:430-4.

9. Gossec L, Smolen JS, Gaujoux-Viala C, Ash Z, Marzo-Ortega H, van der Heijde D, et al. European League Against Rheumatism recommendations for the management of psoriatic arthritis with pharmacological therapies. Ann Rheum Dis 2012;71:4-12.

10. Ash Z, Gaujoux-Viala C, Gossec L, Hensor EM, FitzGerald O, Winthrop K, et al. A systematic literature review of drug therapies for the treatment of psoriatic arthritis: current evidence and meta-analysis informing the EULAR recommendations for the management of psoriatic arthritis. Ann Rheum Dis 2012;71:319-26.

11. Costa L, Del Puente A, Peluso R, Tasso M, Caso P, Chimenti MS, et al. Small molecule therapy for managing moderate to severe psoriatic arthritis. Expert Opin Pharmacother 2017;18:1557-67.

12. Bilal J, Riaz IB, Kamal MU, Elyan M, Sudano D, Khan MA. A systematic review and meta-analysis of efficacy and safety of novel interleukin inhibitors in the management of psoriatic arthritis. J Clin Rheumatol 2018;24:6-13.

13. Coates LC, Helliwell PS. Validation of minimal disease activity criteria for psoriatic arthritis using interventional trial data. Arthritis Care Res 2010;62:965-9.

14. Mease PJ, Heckaman M, Kary S, Kupper H. Application and modifications of minimal disease activity measures for patients with psoriatic arthritis treated with adalimumab: subanalyses of ADEPT. J Rheumatol 2013;40:647-52.
15. Perrotta FM, Marchesoni A, Lubrano E. Minimal disease activity and remission in psoriatic arthritis patients treated with anti-TNF- $\alpha$ drugs. J Rheumatol 2016;43:350-5.

16. Lubrano E, De Socio A, Perrotta FM. Comparison of composite indices tailored for psoriatic arthritis treated with csDMARD and bDMARD: a cross-sectional analysis of a longitudinal cohort. J Rheumatol 2017;44:1159-64.

17. Wofford J, Menter A. Ustekinumab for the treatment of psoriatic arthritis. Expert Rev Clin Immunol 2014;10:189-202.

18. Gottlieb AB, Langley RG, Philipp S, Sigurgeirsson B, Blauvelt A, Martin R, et al. Secukinumab improves physical function in subjects with plaque psoriasis and psoriatic arthritis: results from two randomized, phase 3 trials. J Drugs Dermatol 2015;14:821-33.

19. Keating GM. Apremilast: a review in psoriasis and psoriatic arthritis. Drugs 2017;77:459-72.

20. Coates LC, Navarro-Coy N, Brown SR, Brown S, McParland L, Collier H, et al. The TICOPA protocol (TIght COntrol of Psoriatic Arthritis): a randomised controlled trial to compare intensive management versus standard care in early psoriatic arthritis. BMC Musculoskelet Disord 2013;14:101.

21. Lubrano E, Mesina F, Caporali R. Clinical remission in rheumatoid arthritis and psoriatic arthritis. Clin Exp Rheumatol 2018 Mar 16 (E-pub ahead of print).

22. Schoels M, Aletaha D, Funovits J, Kavanaugh A, Baker D, Smolen JS. Application of the DAREA/DAPSA score for assessment of disease activity in psoriatic arthritis. Ann Rheum Dis 2010; 69:1441-7.

23. Coates LC, Helliwell PS. Defining low disease activity states in psoriatic arthritis using novel composite disease instruments. J Rheumatol 2016;43:371-5.

24. Smolen JS, Schöls M, Braun J, Dougados M, FitzGerald O, Gladman DD, et al. Treating axial spondyloarthritis and peripheral spondyloarthritis, especially psoriatic arthritis, to target: 2017 update of recommendations by an international task force. Ann Rheum Dis 2018;77:3-17.

25. Sterry W, Ortonne JP, Kirkham B, Brocq O, Robertson D, Pedersen $\mathrm{RD}$, et al. Comparison of two etanercept regimens for treatment of psoriasis and psoriatic arthritis: PRESTA randomised double blind multicentre trial. BMJ 2010;340:c147.

26. WINPEPI (PEPI-for-Windows). [Internet. Accessed August 22, 2018.] Available from: www.brixtonhealth.com/pepi4windows.html

27. Strober B, Teller C, Yamauchi P, Miller JL, Hooper M, Yang YC, et al. Effects of etanercept on C-reactive protein levels in psoriasis and psoriatic arthritis. Br J Dermatol 2008;159:322-30.

28. van Mens LJJ, van de Sande MGH, van Kuijk AWR, Baeten D, Coates LC. Ideal target for psoriatic arthritis? Comparison of remission and low disease activity states in a real-life cohort. Ann Rheum Dis 2018;77:251-7.

29. Gossec L, McGonagle D, Korotaeva T, Lubrano E, de Miguel E, Østergaard M, et al. Minimal disease activity as a treatment target in psoriatic arthritis: a review of the literature. J Rheumatol 2018; 45:6-13

30. Wervers K, Vis M, Tchetverikov I, Gerards AH, Kok MR, Appels CWY, et al. Burden of psoriatic arthritis in different definitions of disease activity: comparing Minimal Disease Activity and Disease Activity index for Psoriatic Arthritis. Arthritis Care Res 2018 Apr 2 (E-pub ahead of print). 